\title{
The Comparison of Current Cognitive Status with Cognitive Status in the Last Ten Years among the Elderly with Dementia and Healthy Ones
}

\author{
Sousan Salary ${ }^{1 *}$, Fatemeh Amini², Muhammad Ali Asghari Moghaddam ${ }^{1}$ \\ ${ }^{1}$ Department of Clinical Psychology, University of Shahed, Tehran, Iran \\ ${ }^{2}$ Department of Psychology, Payame Noor University of Lamerd, Lamerd, Iran
}

\begin{tabular}{lll}
\hline & Article Info: & \\
\hline Received: 29 Aug 2016 & Revised: 21 Dec 2016 & Accepted: 28 Jan 2017
\end{tabular}

\section{ABSTRACT}

Introduction: Improvement in health and social situations increased life expectancy. The elderly are more likely experience psychological problems, including loss of cognitive functions. Materials and Methods: The aim of this study was to evaluate current cognitive status among the healthy elderly and those with dementia and to compare that with the recent decade. Accordingly, 230 healthy elderlies from the Jahandidegan center and 98 patients with dementia in Tehran were selected by convenience sampling method. In order to test the status of their current cognitive, the RUDAS and MMSE scales and for the assessment of cognitive status last ten years, IQCDE tool were used. Results: The results showed significant differences between the two healthy and dementia groups in current cognitive status as well as in the last ten years. The mean scores of dementia groups were lower than the healthy group. Conclusion: The results suggest that the elderly with dementia experience significant changes in their cognitive statues and they are faced with effective loss in their cognitive status.

Key words:

1. Aged

2. Patients

3. Dementia

*Corresponding Author: Sousan Salary

E-mail: salary.susan@yahoo.com 
مقايسؤ وضعيت شناختى كنونى با وضعيت شناختى در ده سال اخير ميان سالمندان مبتلا به دمانس و سالمندان سالم دره

سوسن سالارى '":، فاطمه امينى '، محمد على اصغرى مقدم'

اكروه روانشناسى بالينى، دانشكاه شاهد، تهران ،ايران

rكروه روانشناسى، دانشكاه بِيام نور لامرد، لامر د، ايران

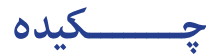

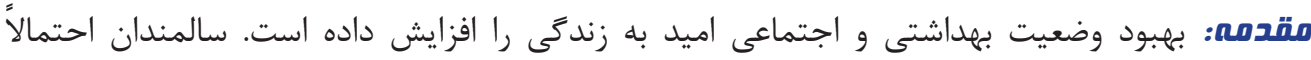

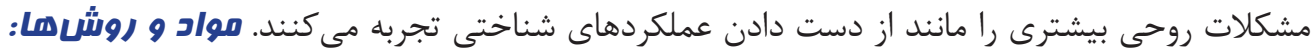

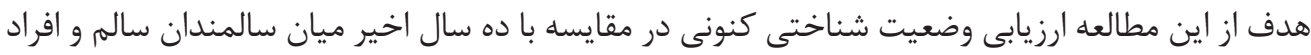

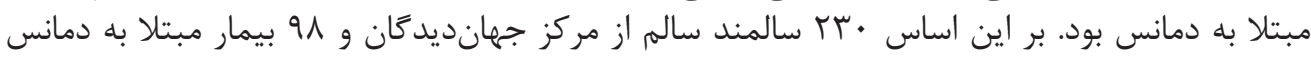

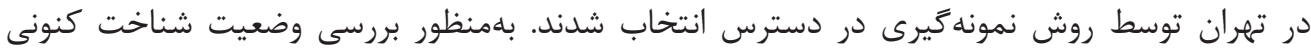

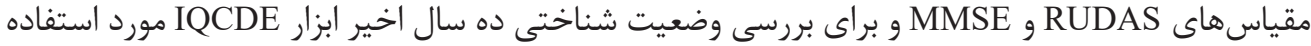

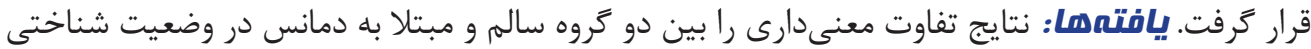

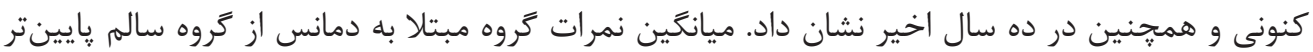

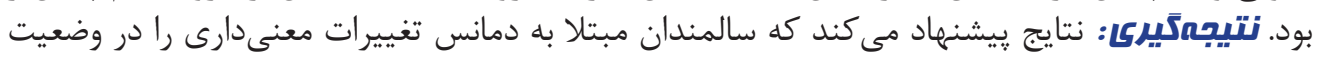

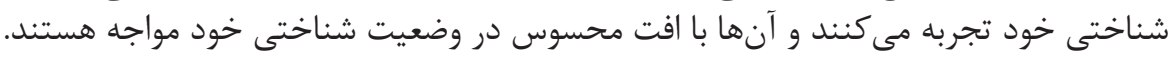

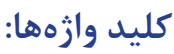
( ب المند

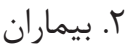

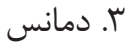




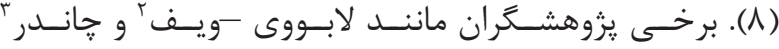

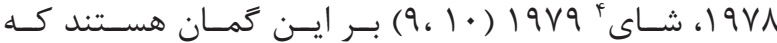

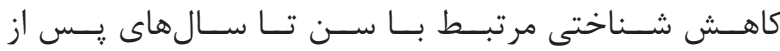

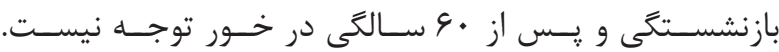

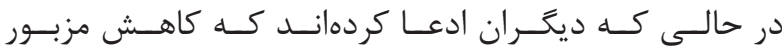

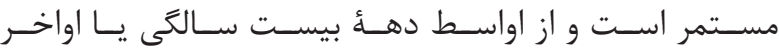

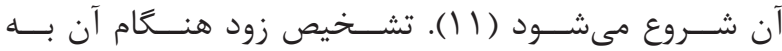

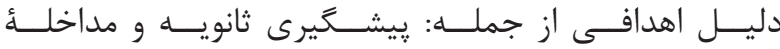

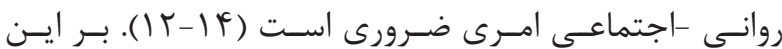

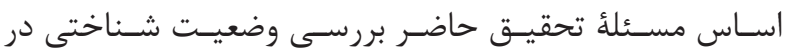

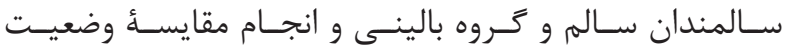

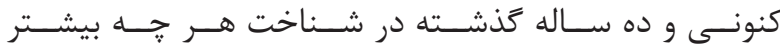

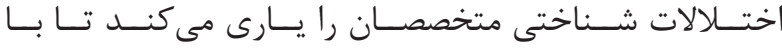

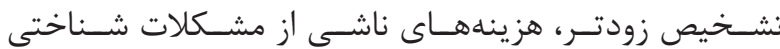

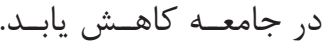

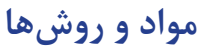

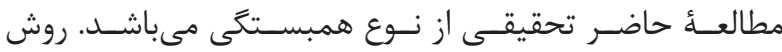

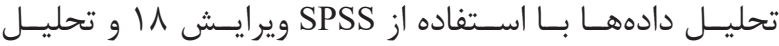

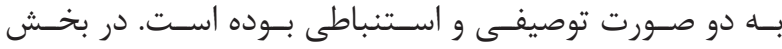

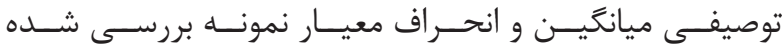

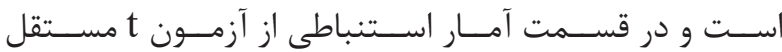

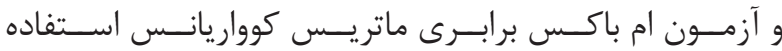

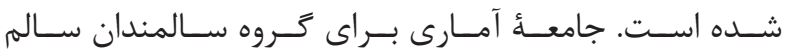

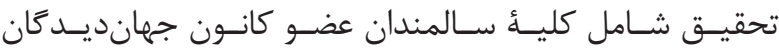

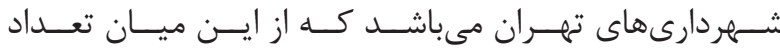

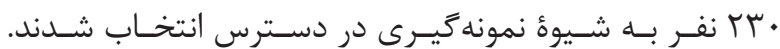

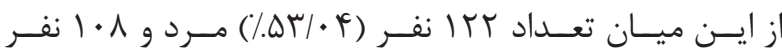

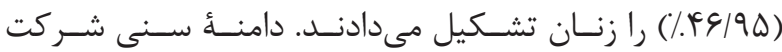

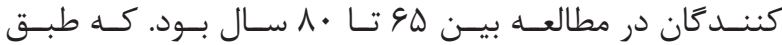

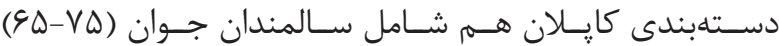

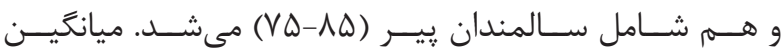

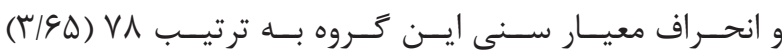

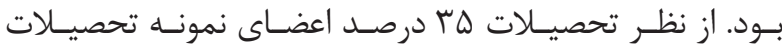

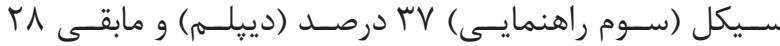

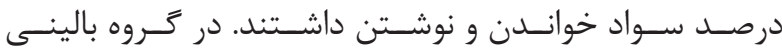

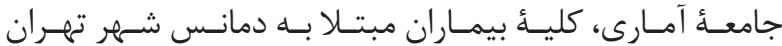

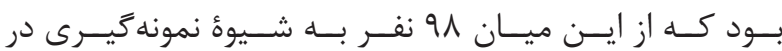

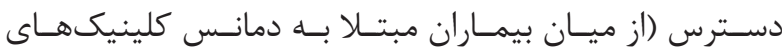

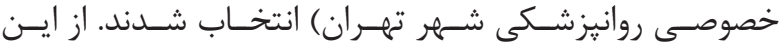
ميـان تعـداد كاع نفـر (

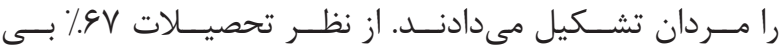

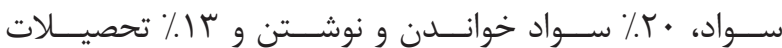

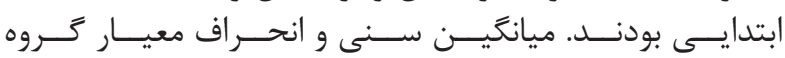

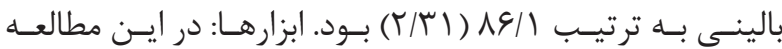

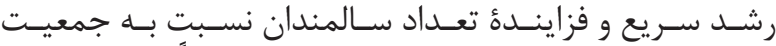

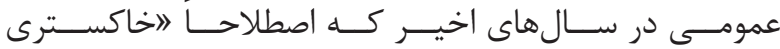

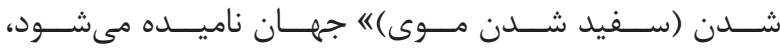

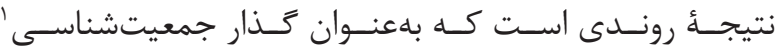

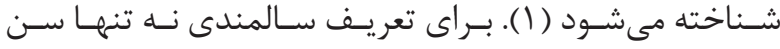

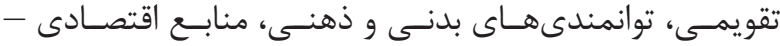

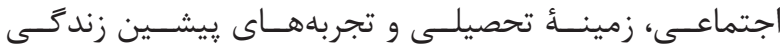

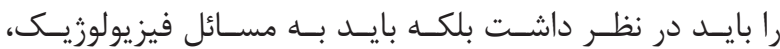

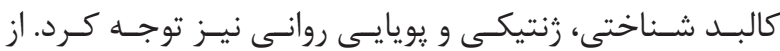

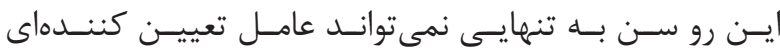

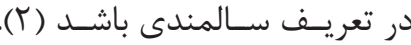

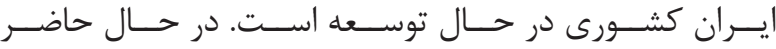

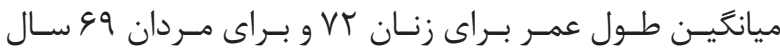

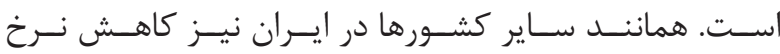

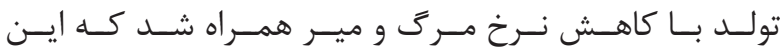

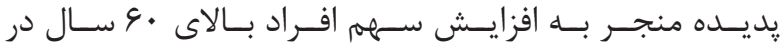

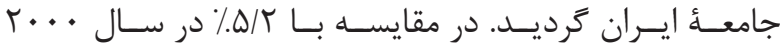

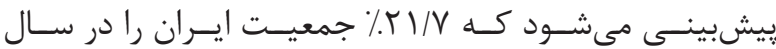

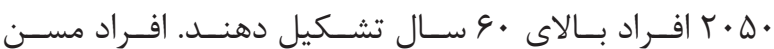

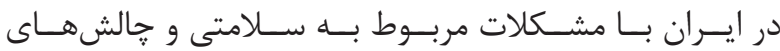

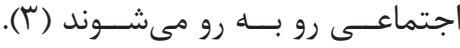

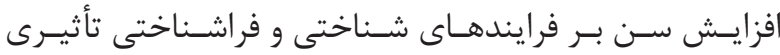

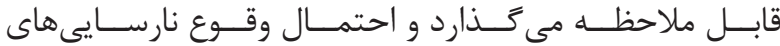

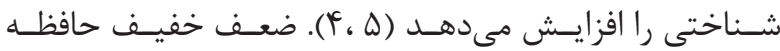

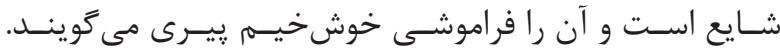

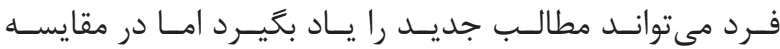

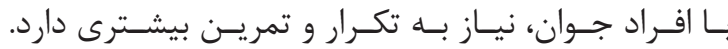

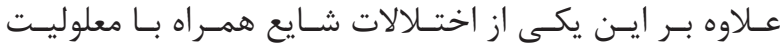

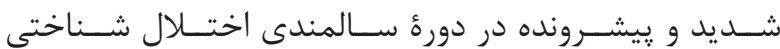

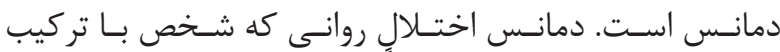

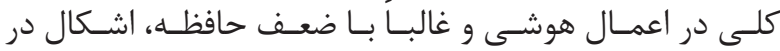

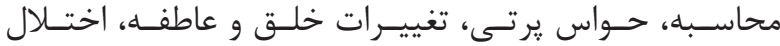

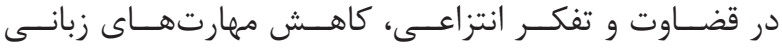

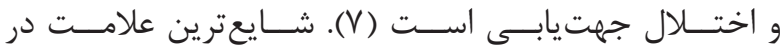

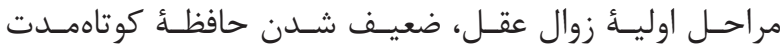

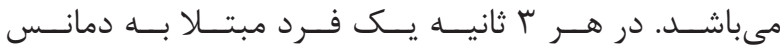

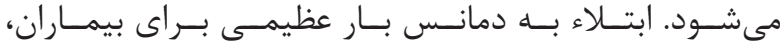

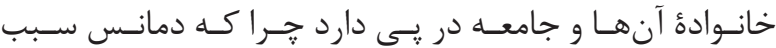

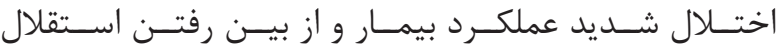

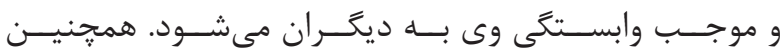

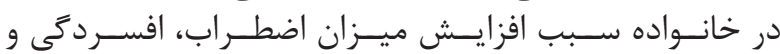

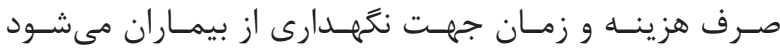 \\ Chandler}




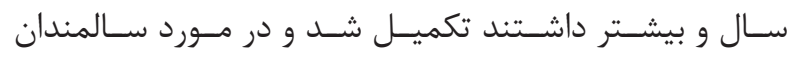

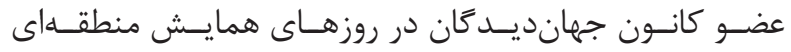

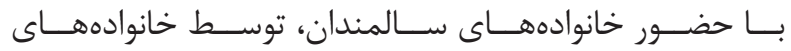

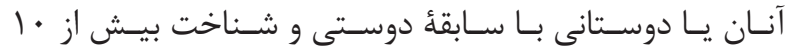

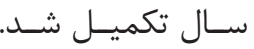

يافتهها

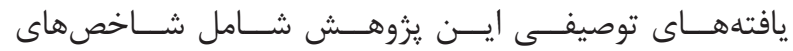

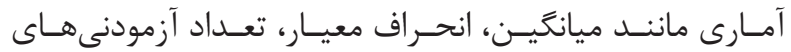

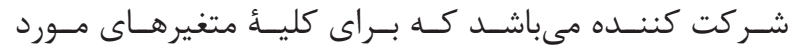

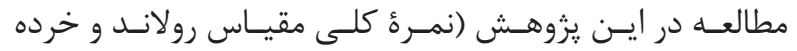

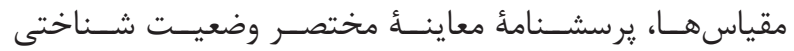

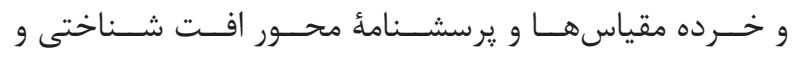

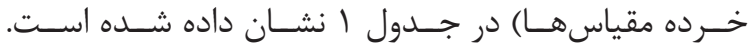

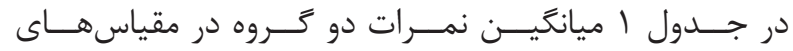

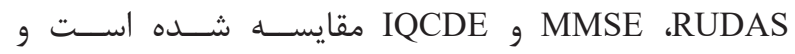

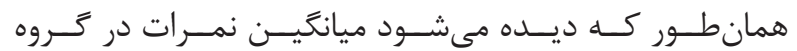

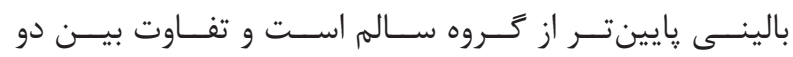

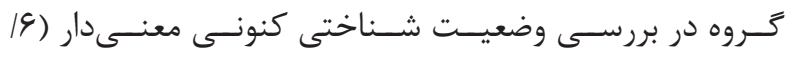
(

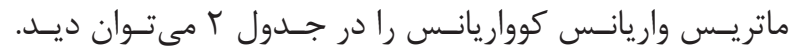

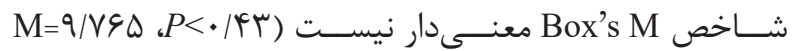

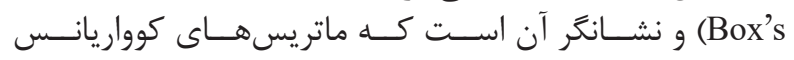

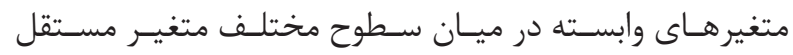

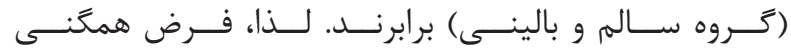

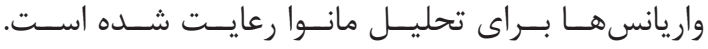

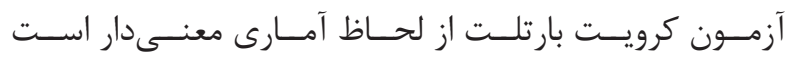

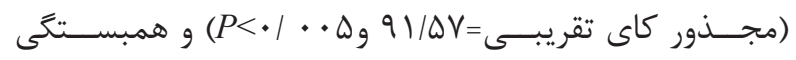

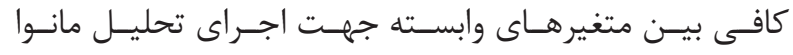

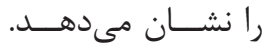

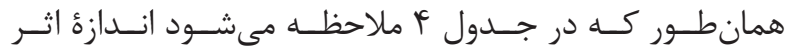

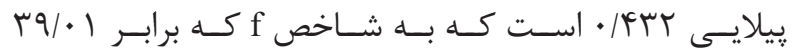

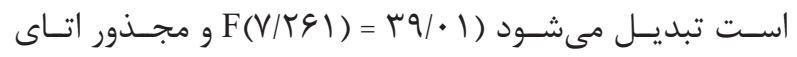

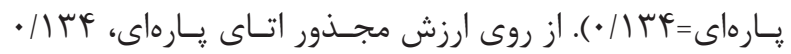

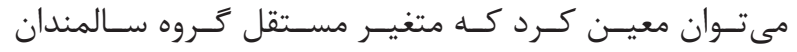

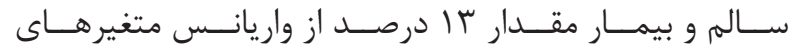

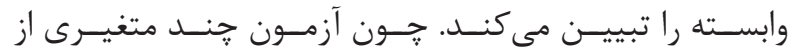

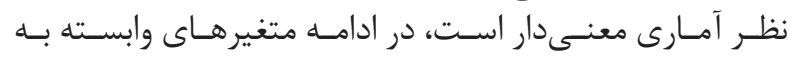

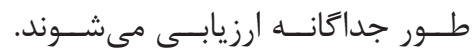

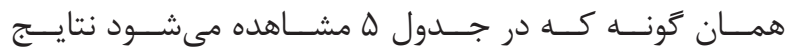

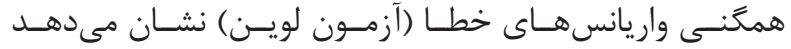

${ }^{5}$ Rowland universal dementia assessment scale

${ }^{6}$ Mini mental state examination

${ }^{7}$ The informant questionnaire on cognitive decline in the

${ }^{8}$ Folstein

${ }^{9}$ Jorm

${ }^{10}$ Episodic memory

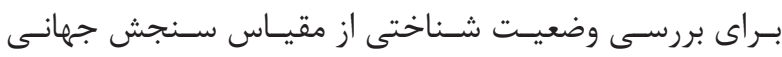

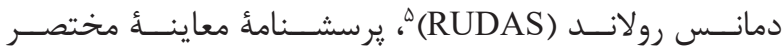

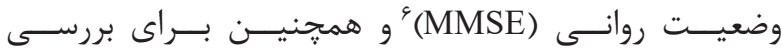

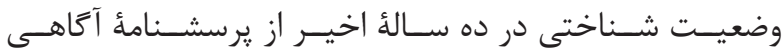

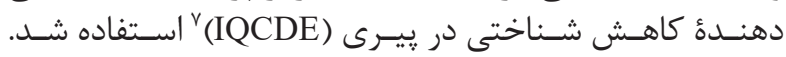

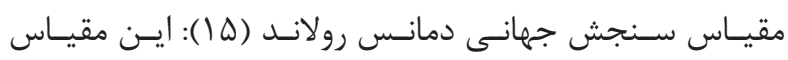

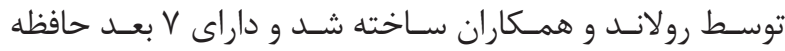

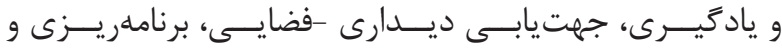

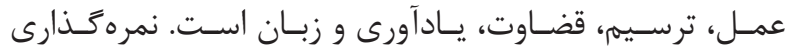

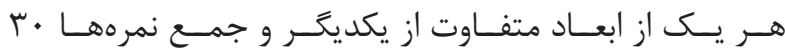

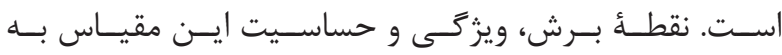

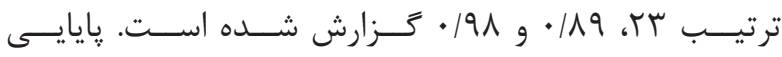

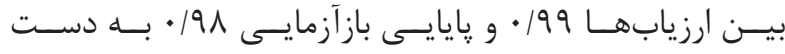

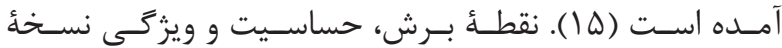

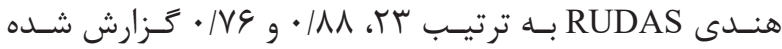

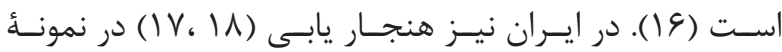

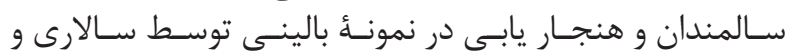

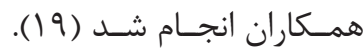

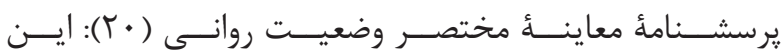

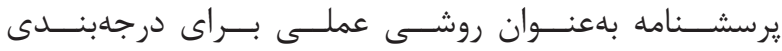

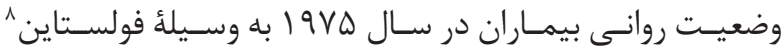

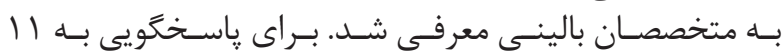

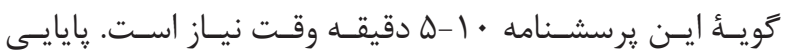

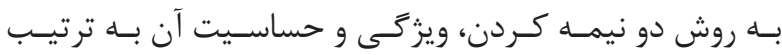

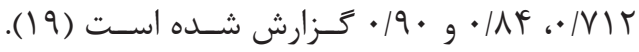

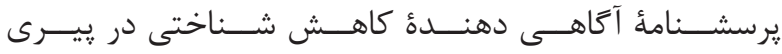

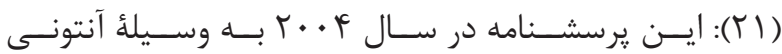

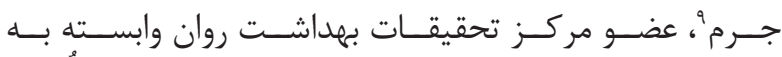

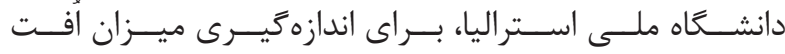

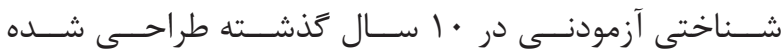

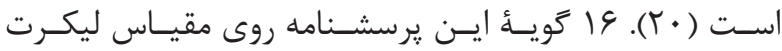

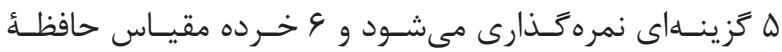

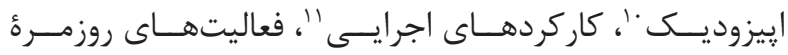

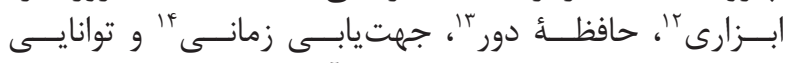

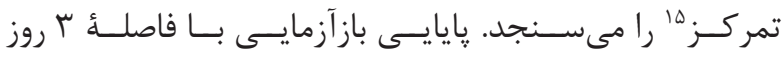

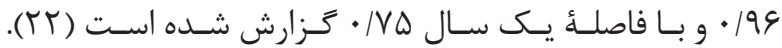

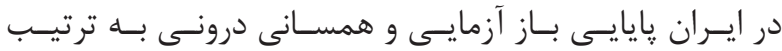

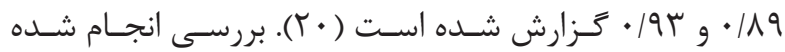

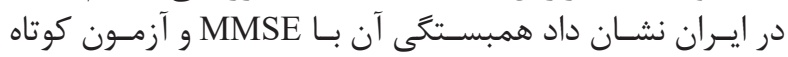

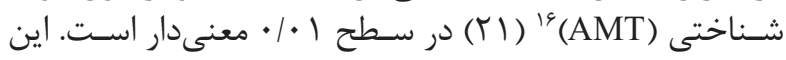

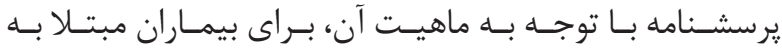

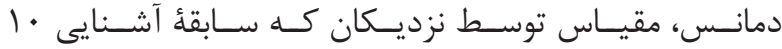

\footnotetext{
${ }^{11}$ Executive functions

${ }^{12}$ Instrumental activities of daily living

${ }^{13}$ Remote memory

${ }^{14}$ Orientation in time

${ }^{15}$ Ability to focus

${ }^{16}$ Abbreviated mental test
} 


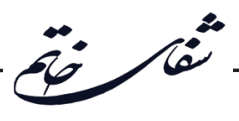

جدول ا- ميانگين، انحراف معيار و تعداد آزمودنى در مقياس RUDAS، MQCDE، و MMSE.

\begin{tabular}{|c|c|c|c|c|c|}
\hline سطام معني، & أثمر الف استباتيلرد & مياثكثئ & Shar & $09 g^{5}$ & متثير \\
\hline \multirow{2}{*}{$+\mid-++1$} & $\mathrm{~T} / \mathrm{V}$ & IF/A & rr. & سالم & \multirow{2}{*}{ تمر \& كل RUDAS } \\
\hline & I/A & p/V & $9 A$ & بالينـى & \\
\hline \multirow{2}{*}{$-1++1 *$} & $r / 1$ & $r / p$ & rr. & سالم & \multirow{2}{*}{ جهنثبايث } \\
\hline & $1 / 1$ & I/T & $9 A$ & يالينتح & \\
\hline \multirow{2}{*}{$+1++t$} & $1 / 9$ & $T / A$ & rr. & سـالم & \multirow{2}{*}{ كار عملي } \\
\hline & $+/ F$ & $1 / \mathrm{V}$ & 91 & يالينـى & \\
\hline \multirow{2}{*}{$-1++1 *$} & +10 & $1 / 9$ & rr. & سالم & \multirow{2}{*}{ تروبهر } \\
\hline & $+/ r q$ & $+/ r$ & $9 \wedge$ & & \\
\hline \multirow{2}{*}{$+1++\infty$} & $r / 1$ & ris & rr. & سالمبم & \multirow{2}{*}{ قغشاوت } \\
\hline & $T / A$ & $r / r$ & 91 & يالينتح & \\
\hline \multirow{2}{*}{$+1++1$} & $r / 1$ & $\Delta / r$ & rr. & سالهم & \multirow{2}{*}{ يادآورى } \\
\hline & $1 / \lambda$ & P/V & qA & بالينـى & \\
\hline \multirow{2}{*}{$+1++1$} & F/r & $\mathrm{v} / \mathrm{I}$ & rr. & سالم & \multirow{2}{*}{ زباث } \\
\hline & $r / r$ & F/s & $9 \wedge$ & يالينـى & \\
\hline \multirow{2}{*}{$+1++1$} & $r / r$ & $\mathbf{V} / \mathbf{A}$ & rr. & سالهم & \multirow{2}{*}{ جالئ إيززوديك } \\
\hline & $r / 1$ & $F / 1$ & 91 & بالينتى & \\
\hline \multirow{2}{*}{$+1+1$} & $r / 1$ & F/r & rr. & سالمر & \multirow{2}{*}{ كاركر دهاى الجرالي } \\
\hline & $1 / 1$ & $r / 1$ & $9 \wedge$ & بالينحى & \\
\hline \multirow{2}{*}{$+1++1$} & $r / T$ & $\Delta / 1$ & rr. & سالم & \multirow{2}{*}{ ثماليتهاى دوزمرة ايثزارى } \\
\hline & $1 / T$ & rir & 91 & يالينـى & \\
\hline \multirow{2}{*}{$+1++1$} & $r / T$ & $9 / 1$ & rr. & سالم & \multirow{2}{*}{ حاف녀 دور } \\
\hline & $1 / T$ & $\mathrm{r} / \mathrm{V}$ & 91 & يالينحى & \\
\hline \multirow{2}{*}{$-1++1 *$} & $r / T$ & $F / 1$ & rr. & سالم & \multirow{2}{*}{ جيثت } \\
\hline & $r / 1$ & r/v & 91 & يالينـى & \\
\hline \multirow{2}{*}{$+1+1$} & r/s & $9 / 1$ & rr. & سالم & \multirow{2}{*}{ توافائى تمركز } \\
\hline & $T / P$ & $\Delta / 1$ & 91 & يالينـى & \\
\hline$+1++1$ & +IVF & FIS & rr. & سالمبم & MMSE 158 mi \\
\hline & $F / 1$ & $\Delta / 1$ & qA & بالينتى & \\
\hline$+1++1$ & $T / r$ & $9 / 1$ & rr. & سالم & M Orientation \\
\hline & $1 / 9$ & F/r & 91 & بالينتى & 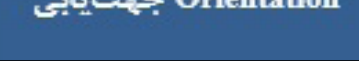 \\
\hline$+1++1$ & r/s & 919 & rr. & سـالهم & Remistration \\
\hline & $T / T$ & $\Delta / r$ & 91 & يالينتح & Kegistration \\
\hline$+1++1$ & r/A & $9 / 1$ & $r r$ & سالمم & Attention Calcnlation \\
\hline & $T / V$ & $\mathrm{r} / \mathrm{T}$ & १人 & يالينتح & Amention Calcukaton \\
\hline$-1+\infty+1$ & ris & $F / 9$ & rr. & سالم & Pecent Memerv \\
\hline & $T / 0$ & T/V & 91 & يالينـت & Recent Memory \\
\hline$+1+0 t$ & $1 / F$ & $8 / 1$ & Tr. & سالهم & Yignonatial Thinlsing \\
\hline & $T / 1$ & $F / l$ & 91 & يالينـق & 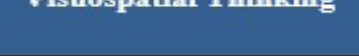 \\
\hline$+1+-1$ & $r / l$ & $\Delta / 1$ & Tr. & سالهم & Trunction \\
\hline & $1 / 0$ & $\mathrm{r} / \mathrm{T}$ & 91 & يالينــ & Function \\
\hline
\end{tabular}




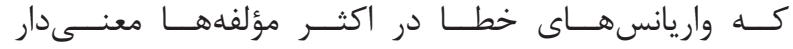

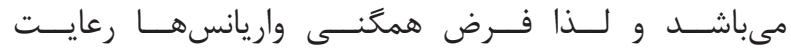

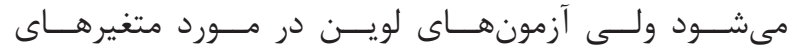

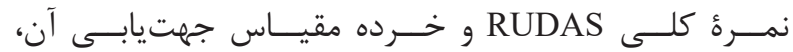

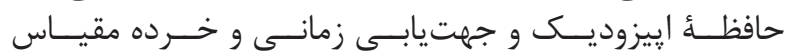

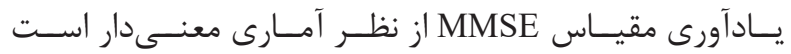

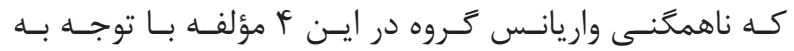

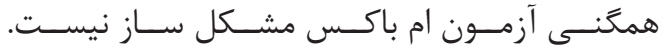

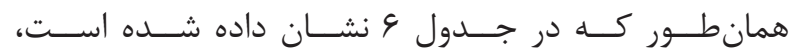

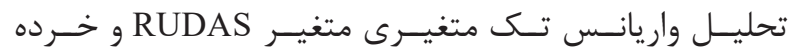

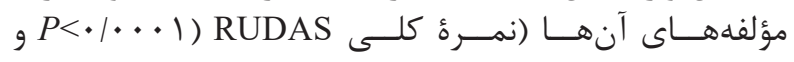

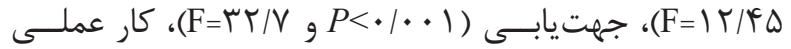
(F=

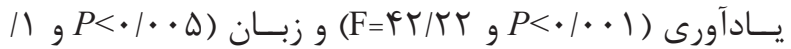

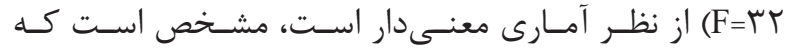

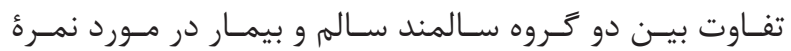

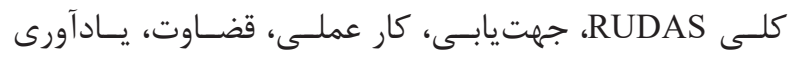

جدول r- خلاصُٔ نتايج آزمون ام باكس برابرى ماتريس كوواريانس.

\begin{tabular}{|c|c|c|c|c|}
\hline سطح معنى دارى & df 2 & df 1 & f & ام باكس \\
\hline . pr & TrI/I & $\Lambda$ & $1 / 1 F$ & $q / V \varphi \Delta$ \\
\hline
\end{tabular}

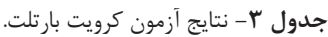

\begin{tabular}{|c|c|c|c|}
\hline سطح معنى دارى & $\mathrm{df}$ & مجذور خى تقريبى & نسبت احتمالى \\
\hline$P<\cdot 1 \cdot \Delta$ & $\Lambda$ & $91 / 0 \mathrm{~V}$ & $<\cdot \cdots \theta$ \\
\hline
\end{tabular}

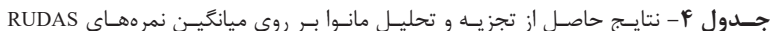

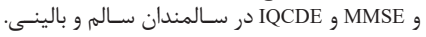

\begin{tabular}{|c|c|c|c|c|c|c|}
\hline مجلذر: التاى بارهاى & سطح منىى فارى & خ df & فرضيه & $\mathrm{F}$ & هقدار & نام أهمون \\
\hline . $1 \pi \mathrm{F}^{-}$ & $P<\cdot|\cdots|$ & $r y \mid$ & y & $r q / .1$ & 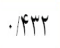 & اثر بيالايى \\
\hline · $1 \pi r$ & $P<\cdot \ldots 1$ & tol & y & $r q / .1$ & . & لامبداى ويلكز \\
\hline . & $P<\cdot \ldots\}$ & $\mu \varphi$. & y & $r q / .1$ & N NT & أر هتلينك \\
\hline - TYA & $P<., \ldots 1$ & YM & $\Delta$ & $r q / .1$ & - MIT & يرز كَترين ريشه روى \\
\hline
\end{tabular}

جدول ه- نتايج همَنى واريانسهاى خطا (آزمون لوين).

\begin{tabular}{|c|c|c|c|c|}
\hline سطاب ععثى داري & df 2 & df 1 & f & مئيt \\
\hline+1.49 & TIT & $\wedge$ & $F / 90$ & نمرة كل \\
\hline$* 1 * \theta$ & TIT & $\wedge$ & r/AF & جهت يائى \\
\hline.$/ T F$ & TIT & $\wedge$ & $\Delta / 91$ & كار عملى \\
\hline$+1 . \vee 9$ & TIT & $\wedge$ &.$/ 1 \mathrm{rF}$ & ترسيتم: \\
\hline.$/ 179$ & TIT & $\wedge$ & r/or & قداوت \\
\hline $4 / 4$ & TIT & $\wedge$ & r/VA & 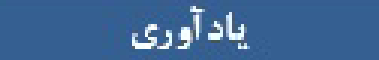 \\
\hline .190 & TIT & $\wedge$ & $\mathrm{V} / \mathrm{rt}$ & 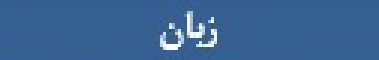 \\
\hline$* 1 * \omega$ & TIT & $\wedge$ & $\Delta / 91$ & 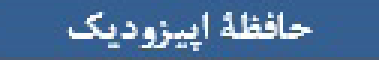 \\
\hline$*|\mathrm{~V}|$ & TIT & $\wedge$ & $F(T)$ & كاركردهاى اجبرائ \\
\hline +/Irt & TIT & $\wedge$ & $9 / T)$ & فعاليتهاى ورزمرة البوارى \\
\hline$* / \Delta F$ & TIT & $\wedge$ & $f(\Delta)$ & موان \\
\hline$+1 \cdot \mathrm{Fr}$ & TIT & $\wedge$ & FITF & 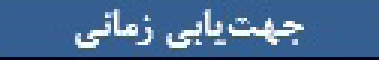 \\
\hline.$|9|$ & TIT & $\wedge$ & P/rr & تواتائ تعركز \\
\hline .199 & TIT & $\wedge$ & Q/fr & نمرة كل \\
\hline $4 / \mathrm{V} 9$ & TIT & $\wedge$ & $9 / 11$ & جيثت بائي \\
\hline .190 & TIT & $\wedge$ & DIST & كار عملى \\
\hline$+|A|$ & TIT & $\wedge$ & $r / P 1$ & تربئما \\
\hline$+/ T I+$ & TIT & $\wedge$ & $\Delta / T r$ & قداوت \\
\hline$* 1 *+r$ & TIT & $\wedge$ & G/TT & ياد آورى \\
\hline.$/ 1 T$ & TIT & $\wedge$ & $0 / 99$ & زئن \\
\hline
\end{tabular}




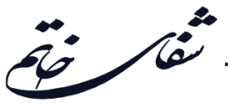

جدول צ- نتايج حاصل از تحليل واريانس يك راهه در متن مانوا بر ميانَين نمرءٔ كلى RUDAS و MMSE دQCDE در سالمندان سالم و بالينى و مؤلفههاى آن.

\begin{tabular}{|c|c|c|c|c|c|c|}
\hline 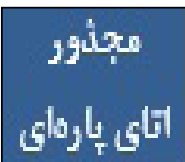 & $4 \sin$ & $F$ & 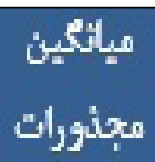 & 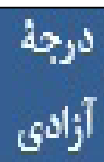 & Egpr & مبتري \\
\hline$+\| T$ & $+\mid+++1$ & $\pi / f 0$ & TTAITA & 9 & $\mathrm{FAF} / \mathrm{YF}$ & ثرة: \\
\hline$+/ 1 \vee \psi$ & $+|++|$ & THIV & $\mid F_{*} / 4$ & 4 & TTI/A & $4 t^{4}+4$ \\
\hline$+/ 1 \Delta \gamma$ & $+1+t$ & $T / 1 T$ & $11 / F$ & 9 & $T T / A$ & ther \\
\hline$+1+f f$ &.$/ F T$ & $11 / T$ & 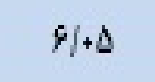 & 9 & $1 T / 1$ & Anty \\
\hline.$+\% D$ & $+\mid+*+1$ & $\Delta F / f t$ & $T V / / A * \Delta$ & 9 & $\Delta F T / P]$ & 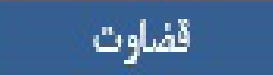 \\
\hline$+/ 1 f A$ & $+1++1$ & FF/T & TAT/F & 9 & $Y_{\Delta} / \mathrm{T}$ & $6 y]^{15}$ \\
\hline+114 & $+1+4$ & $\mathrm{Tth}$ & IVT/To & 9 & TFF/A & 54 \\
\hline$+/ 1 T T$ & $+1+\infty$ & frit" & $|\Delta \tilde{F}| \mid$ & 4 & $T H / T$ & 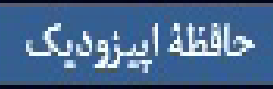 \\
\hline$+1+4 \mathrm{~A}$ & $+1++y$ & $\Delta M T$ & TTV/10 & 9 & PSFIT & (5) \\
\hline$+|++|+$ & $+\mid+*+1$ & THIV & $T \Delta 1 /+\Delta$ & 9 & $\Delta+T / 1$ & 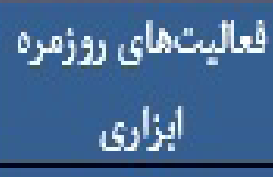 \\
\hline$+/ 11 \mathrm{Y}$ & $+4+1$ & $V F / T$ & $T / F / 1$ & 9 & Frtit & yt ditu \\
\hline$+1+V F$ & $+1+++1$ & $T T / 9$ & $|\mathrm{Y}|=\Delta$ & 9 & $\mathrm{TFthl}$ & 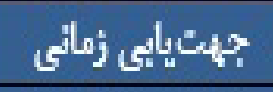 \\
\hline$+/ 10 T$ & $+1++\%$ & $\mathrm{Y} / \mathrm{A \Delta}$ & TV/ffo & 9 & $\Delta F I A 9$ & 5 Fin \\
\hline$+1+++1$ & $-\mid++T H$ & TMY & rif/vis & 9 & YAVIfT & $5^{5}$ \\
\hline$+\| F+$ & $+\mid+++1$ & $F \| / \mathrm{Y}$ & $T \mathrm{TTT} /++\Delta$ & 9 & $\Delta f f /+1$ & 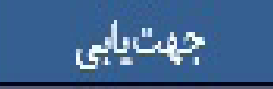 \\
\hline$+F \Delta$ & $+1++T$ & $T / T$ & $10 / T+T$ & 1 & $T / F+F$ & story \\
\hline$+/ 1 Y V$ & $+4++i$ & $\mathrm{~V} / \mathrm{Fi}$ & $Y \varphi_{\Delta / 1+F}$ & i & $\Delta T / T I T$ & ptow \\
\hline$+1+\Lambda \Delta$ & $+\mid++1$ & $+F / H$ & $\dot{F}_{\Delta / T} \hat{F}_{+}$ & i & $T / \Delta T H$ & 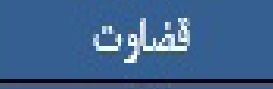 \\
\hline$+/ 1 / 19$ & 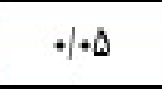 & $\mathrm{F} / \mathrm{A}$ & Fo/ITT & 1 & $T / T F_{B}$ & $6 y]^{1 / 4}$ \\
\hline$+1+1+$ & $++*+1$ & F/T & $\mathrm{F} \Delta / \mathrm{TH}$ & 1 & $\mathrm{~W} / \mathrm{HA}$ & 45 \\
\hline
\end{tabular}

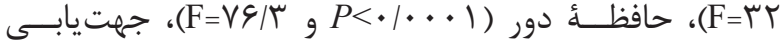

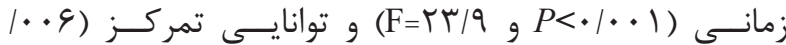

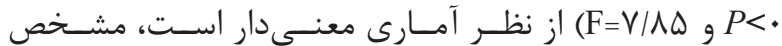

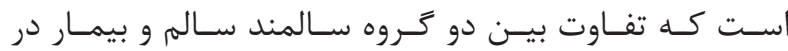

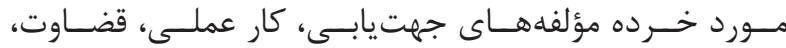

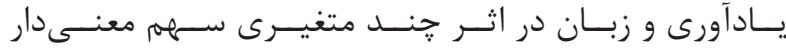

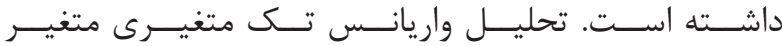

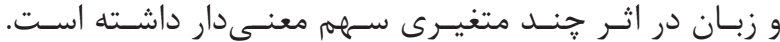

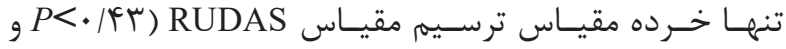

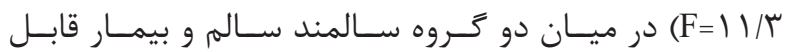

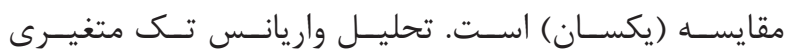

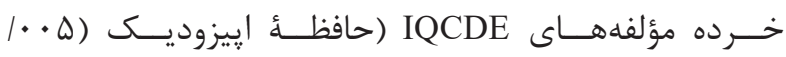

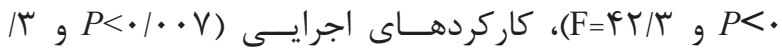
IV 


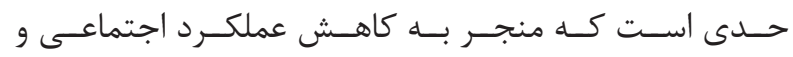

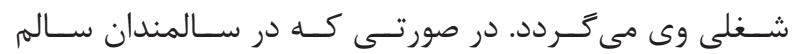

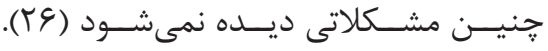

در مــورد وضعيــت شــناختى كنونــى دو مقيــاس اســتفاده

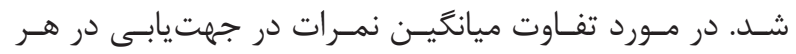

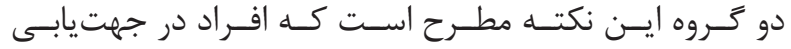

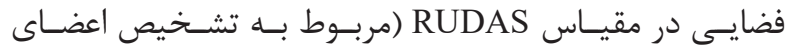

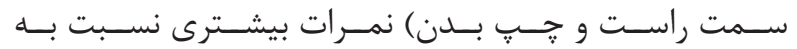

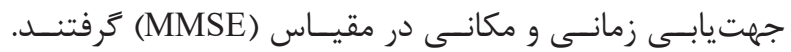

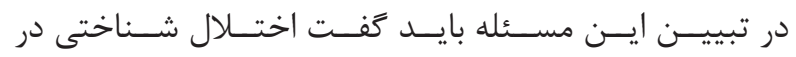

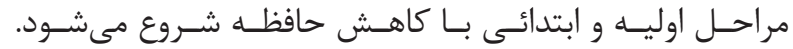

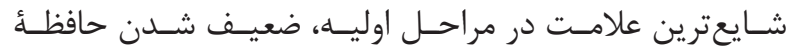

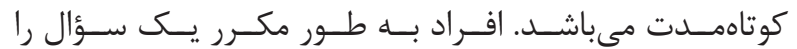

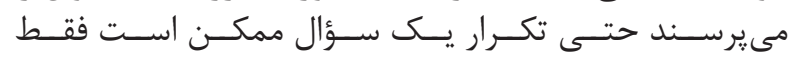

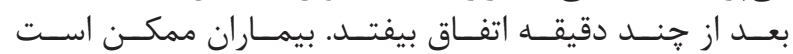

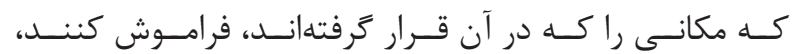

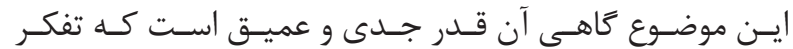

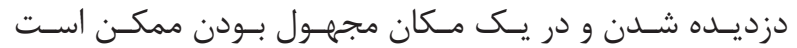

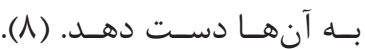

در يـك جمعيـت طبيعىى، بـا افزايش سـن، كاهـش در عملكرد

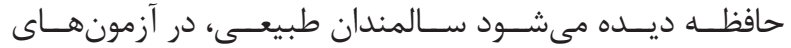

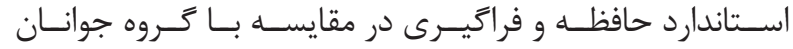

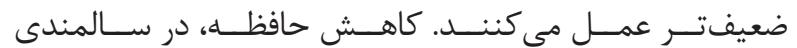

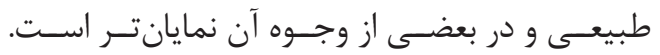

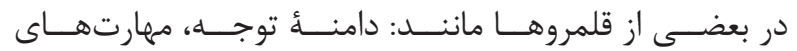

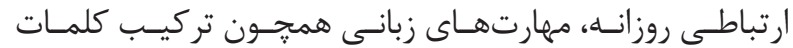

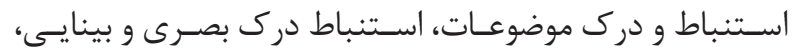

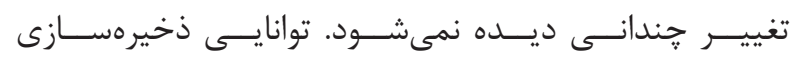

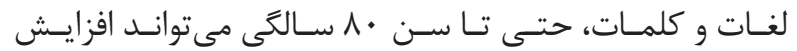

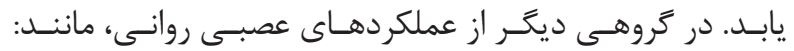

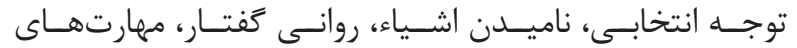

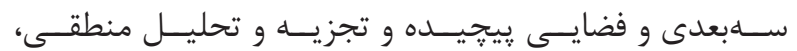

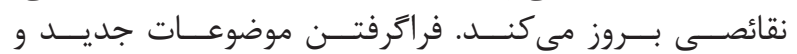

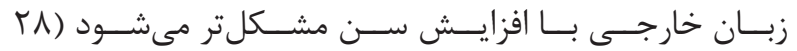
كاهو، (YV،

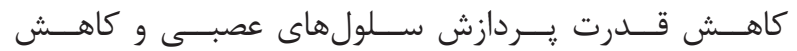

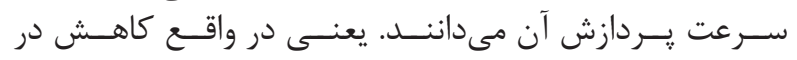

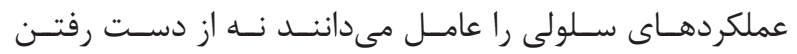

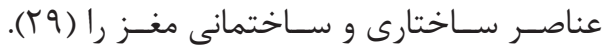

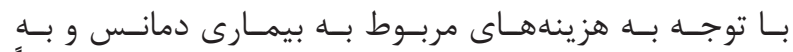

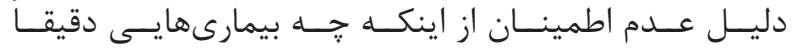

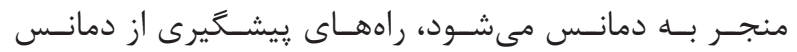

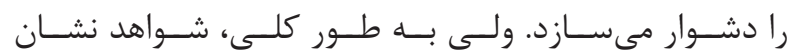

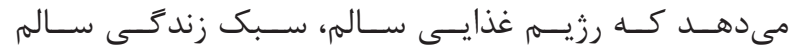

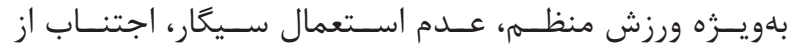

${ }^{17}$ Peripheral

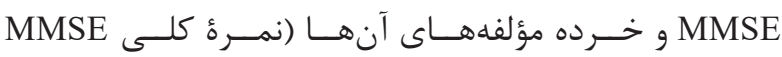

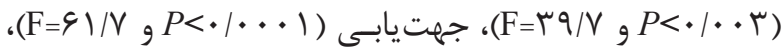
كار عملـى (r) ( F

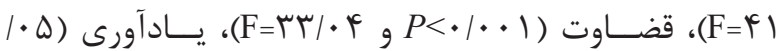

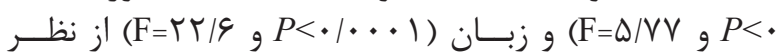

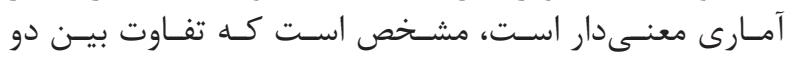

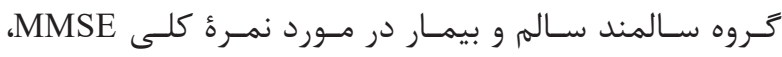

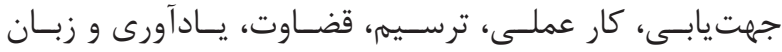

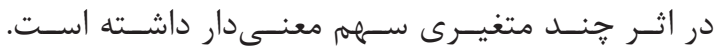

بحث و نتيجه كيرى

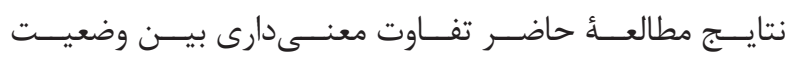

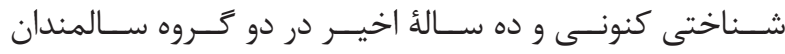

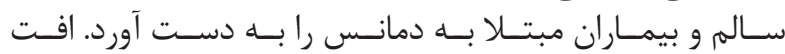

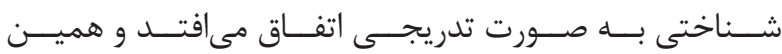

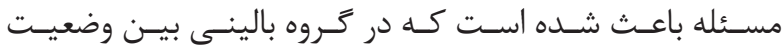

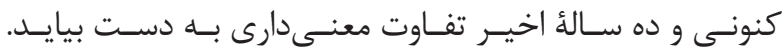

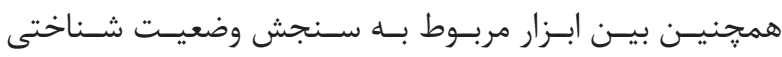

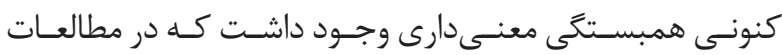

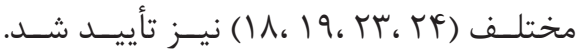

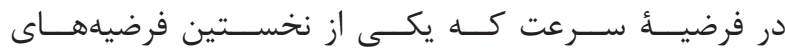

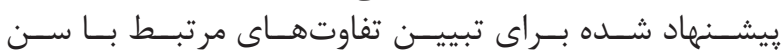

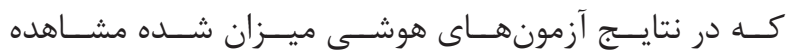

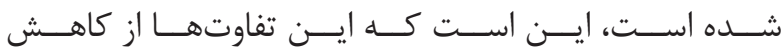

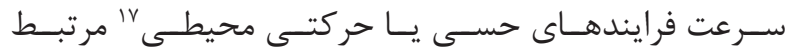

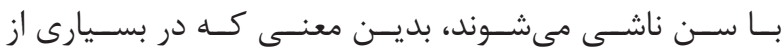

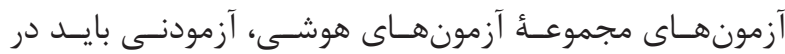

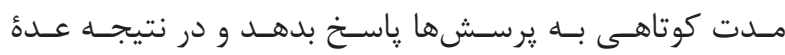

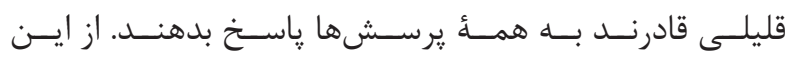

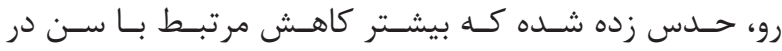

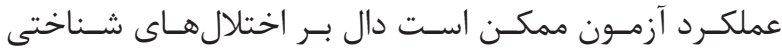

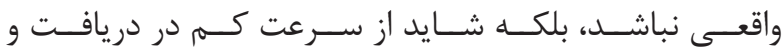

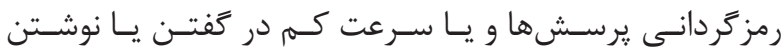

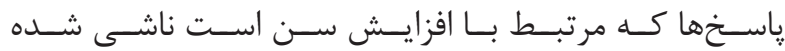

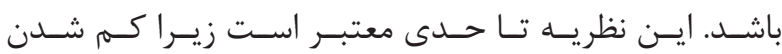

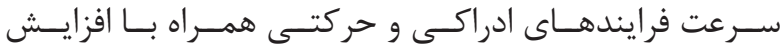

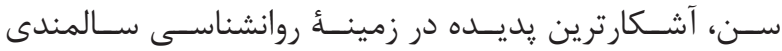

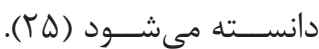

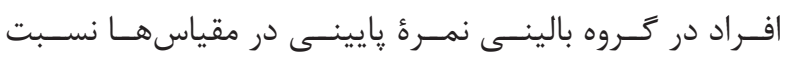

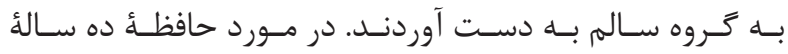

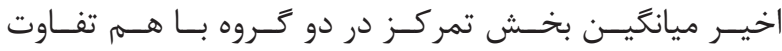

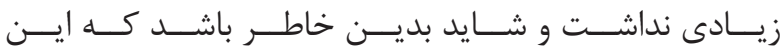

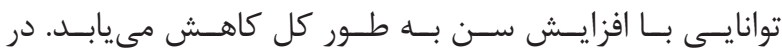

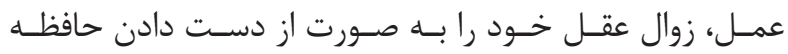

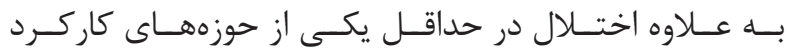

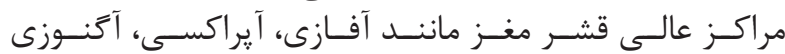

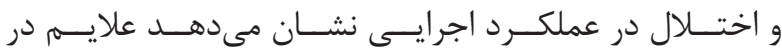




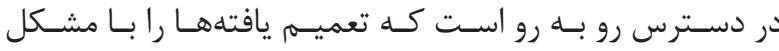

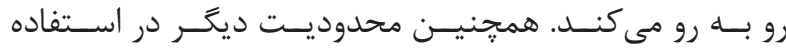

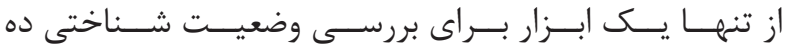

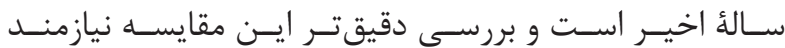

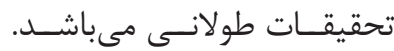

1. World Health Organization. "Social development and ageing: crisis or opportunity?" Special panel at Geneva. Switzerland. 2000; p. 4.

2. Jarvik LF, Small GW. Geriatric psychiatry: introduction. Sadock BJ, Sadock VA. Comprehensive textbook of psychiatry. $7^{\text {th }}$ ed. Philadelphia: Lippincott Williams \& Wilkins. 2000.

3. Kiani S, Bayanzadeh M, Tavallaee M, Hogg RS. The Iranian population is graying: are we ready? Arch Iran Med. 2010; 13(4): 333-9.

4. Craik IM, Salthouse ST, Mahwah, NJ. The handbook of aging and cognition. $2^{\text {nd }}$ ed. US: Lawrence Erlbaum Associates. 2000.

5. Dixon R, Backman L, Nilsson LG. New frontiers in cognitive aging. England: Oxford University Press. 2004.

6. Kaplan H, Sadocks V. Handbook of psychiatry behavioral and sciences - clinical psychiatry. $3^{\text {th }}$ ed. Tehran: Shahre ab. 2005.

7. Chop WC, Robnett HR. Gerontology for the health care from fissional. Philadelphia: FA Davis Company. 1999.

8. Fallahi-khoshkanad M, Mazaheri M, Nazari SH. Dementia care and rehabilitation. Tehran: Resaneye Takhasosi. 2009.

9. Labouvie ViefG. Chandra MJ. Cognitive development and lifespan developmental theory: idealistic versus conceptualistic perceive. P.B.Baltes.life -span developmental and behavior. New York: Academic Press. 1987; p. 181-210.

10. Schaie KW. The primary mental abilities in a adulthood: an exploration of psychometric intelegence. Baltes PB, Brim OG. Life-span developmental and behavior. New York: Academic Press. 1979.

11. Wolf-Klein GP, Silverstone FA, Levy AP, Brod MS. Screening for Alzheimer's disease by clock drawing. J Am Geriatr Soc. 1989; 37(8): 730-4.

12. Small GW. Differential diagnosis and early detection of dementia. American Journal of Geriatric Psychiatry.

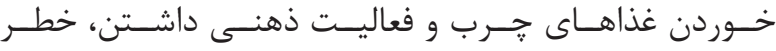

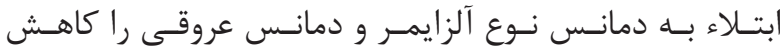

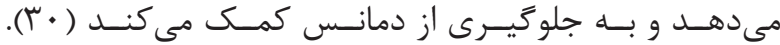

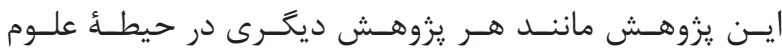

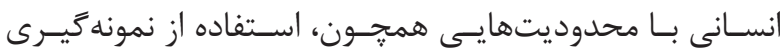

منابع

1998; 6(2): 26-33.

13. Sandson T, Price B. Diagnostic testing and dementia. Neurologic Clinics. 1996; 14(1): 45-59.

14. Brodaty H, Moore CM. The clock drawing test for dementia of thealzheimer's type: a comparison of three scoring methods in a memory disorders clinic. International Journal of Geriatric Psychiatry. 1997; 12(6): 619-27.

15. Storey JE, Jeffrey TJ, Rowland DA, Conforti G, Hugh G, Dickson R. The rowland universal dementia assessment scale (RUDAS): a multicultural cognitive assessment scale. Int Psychogeriatr. 2004; 16(1): 13-31.

16. Iype T, Ajitha BK, Antony P, Ajeeth NB, Job S, Shaji KS. Usefulness of the rowland universal dementia assessment scale in South India. J Neurol Neurosurg Psychiatry. 2006; 77(4): 513-4.

17. Salari S, Shaeiri M, Asghari-Moghadam MA. Validity and reliability the rowland universal dementia assessment scale in a sample of elderly women. Iranian Journal of Ageing. 2013; 8(30): 63-73.

18. Salari S, Shaeiri M, Asghari-Moghadam MA. Assessing the validity and reliability of universal dementia assessment scale (RUDAS) in patients with dementia. Zahedan J Res Med Sci (ZJRMS) 2013; 15: 29-31.

19. Salari S, Shaeiri M, Asghari-Moghadam MA, Masomiyan S. Psychometric characteristics of the rowland universal dementia assessment scale amongst Iranian elderly. Journal of Research and Health. 2015; 5(2): 127-33.

20. Foroughan M, Jafari Z, Shirinbayan PF, Barahani ZR, Rahgozar M. Standardization mini mental status examination (MMSE) in elderly in Tehran. J New Cogn Sci. 2009; 10(2): 29-37.

21. Jorm AF, Jacomb PA. The informant questionnaire on cognitive decline in the elderly (IQCDE): sociodemographic correlates, reliability, validity and some norms. Psychol Med. 1989; 19(4): 1015-22.

22. Allain TJ, Wilson AO, Gomo ZA, Adamchak DJ, Matenga JA. Abbreviated mental test (AMT) in the 
elderly: shortcoming of an adapted AMT in Zimbabwe. Cent Afr J Med. 1996; 42(4): 98-101.

23. Basic D, Khoo A, Conforti D, Rowland J, Vrantsidis F, Logiudice D, et al. Rowland universal dementia assessment scale, mini-mental state examination and general practitioner assessment of cognition in a multicultural cohort of community-dwelling older persons with early dementia. Australian Psychologist. 2006; 44(1): 40-53.

24. Ismail Z, Rajji TK, Shulman KI. Brief cognitive screening instruments: an update. Int $\mathrm{J}$ Geriatr Psychiatry. 2010; 25(2): 111-20.

25. Sandson T, Price B. Diagnostic testing and dementia. Neurologic Clinics. 1996; 14: 45-59.

26. McClane, Kimberly S. Screening instrument for use in complete geriatric assessment. Clinical Nurse
Specialist. 2006; 20(4): 201-7.

27. Hazzard WR, Blass JP, Ettinger WH, Halter JB, Ouslader JG. Principles of geriatric medians and gerontology. $4^{\text {th }}$ ed. New York: Mc Graw -Hill. 1999; p. 225-6.

28. Beers Mark H, Berkow R. Aging and mental health. The merck manual of geriatrics. $3^{\text {th }}$ ed. USA: Merk. 2000. p.307-10.

29. Barry S, Fogel MD, Randolph B, Schiffer MD, Stephen M. Synopsis of neuropsychiatry. $1^{\text {st }}$ ed. USA Lippincott Williams Wilkins(LWW). 2000; p. 528.

30. Iliffe S, Wilcock J, Griffin M, Jain P, Thuné-Boyle $\mathrm{I}$, Koch $\mathrm{T}$, et al. Evidence-based interventions in dementia: a pragmatic cluster-randomised trial of an educational intervention to promote earlier recognition and response to dementia in primary care (EVIDEMED). Terials. 2010; 13(11): 1-10. 